\title{
A robust and accurate segmentation of the knee bones from CT data
}

Ringenbach, Alex ${ }^{1} /$ Schwägli, Tobias ${ }^{2}$

${ }^{1}$ Institute for Medical and Analytical Technologies, University of Applied Sciences Northwestern Switzerland, CH4132 Muttenz, Switzerland, alex.ringenbach@fhnw.ch

${ }^{2}$ Medivation AG, Dorfstrasse 69, CH-5210 Windisch, Switzerland, tobias.schwaegli@medivation.ch

\begin{abstract}
For the production of cutting blocks for knee-joint implant positioning a precise segmentation of the femur and tibia is essential. Due to low bone density and osteophytes the segmentation of knee bones from CT data can be a major challenge. As part of an industrial project, we have developed a hybrid segmentation method - based on a presegmentation with statistical shape model and a fine-segmentation with the Fast Marching algorithm.
\end{abstract}

\section{Introduction}

For the accurate implant positioning of a knee-joint replacement there are more and more custom-made cutting blocks used, which gives the surgeon the necessary bone interface. The advantage of this method over a conventional surgery is the more simple alignment of the implants, a shorter operation time as well as reduced instruments [1] [2].

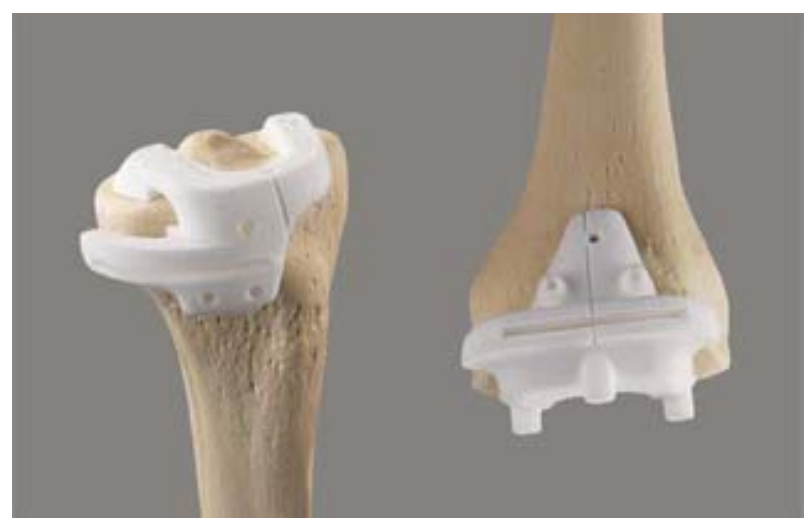

Image 1 Custom made cutting blocks at the bone model (left: tibia, right: femur).

The core for such a technology is a high-precision segmentation of the femur and tibia in the knee joint region (i.e. generation of surface data), as well as the knowledge of the position of the knee, hip and ankle. Our goal is to automate this process as much as possible. In the following we focus on the aspect of robust and accurate segmentation of the knee-bones.

Generally, the segmentation of bones in CT data isn't a particular challenge. However, in image data - due to a knee replacement - the bone segmentation is due to low bone density and osteophytes a challenging problem (see
Image 2), which we solve with a hybrid segmentation approach - containing a pre- and a fine-segmentation.

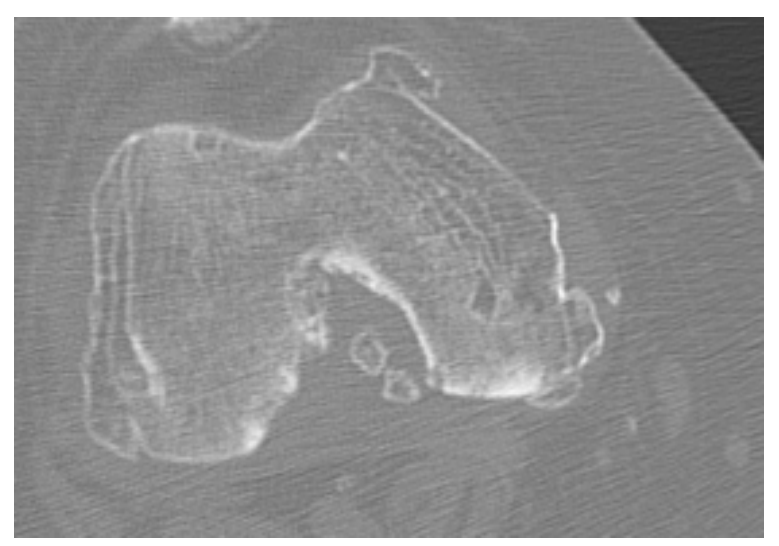

Image 2 CT slice image of the femur: the bone voxelvalues are hardly different from those of the soft tissue.

For the detection of hip, knee and ankle (and hence the information of the leg axis) we use a statistical shape model (based on few data sets) and match it with the CT data. With that, a pre-segmentation of the knee joint bones is given, which guarantees (due to the model-based approach) a robust separation of the femur, tibia and patella. But for the manufacturing of cut-blocks these surface data are not accurate enough - patient-specific characteristics, such as osteophytes, are rarely recorded. To capture the local shape details as well as possible, we perform a further step, a fine segmentation (in the boundary segment region) by using the front-propagation method Fast Marching. This process step can also be applied only locally, i.e. at the support surfaces of the cutting blocks.

\section{Methods}

Our approach of the knee joint segmentation is essentially done in two steps: First, we perform a pre-segmentation 
with the goal to identify and separate the bones robustly, and then a fine segmentation (in the boundary segment region) to detect the local nuances and osteophytes on the bones as accurately as possible.

\subsection{Pre-Segmentation}

The goal of the pre-segmentation is a robust identification and separation of the knee bones: femur, tibia and patella. Especially in case of joint wear and tear (osteoarthritis knee) is a separation of the femur and Tiba (only on the basis of the voxel values) hardly possible - a-priori knowledge is necessary. To implement this step, we use a statistical shape model and match it with the CT data.

\subsubsection{Statistical shape model}

To generate the statistical shape model, we have manually segmented femur, tibia and patella from 30 CT data sets and calculated from these (through correspondence and principal component analysis) the average models and their basic shape variations [3] [4].
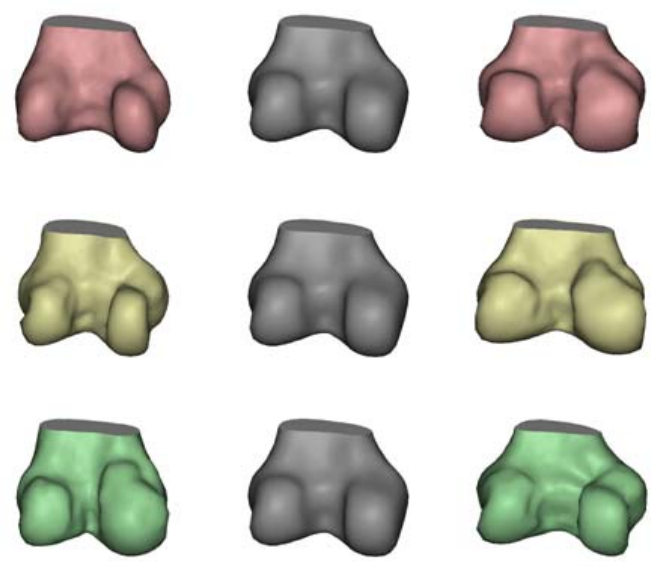

Image 3 Statistical shape model of the femur in the kneearea: Line 1 to 3 shows the first 3 variations with $\pm 3 \sigma$.

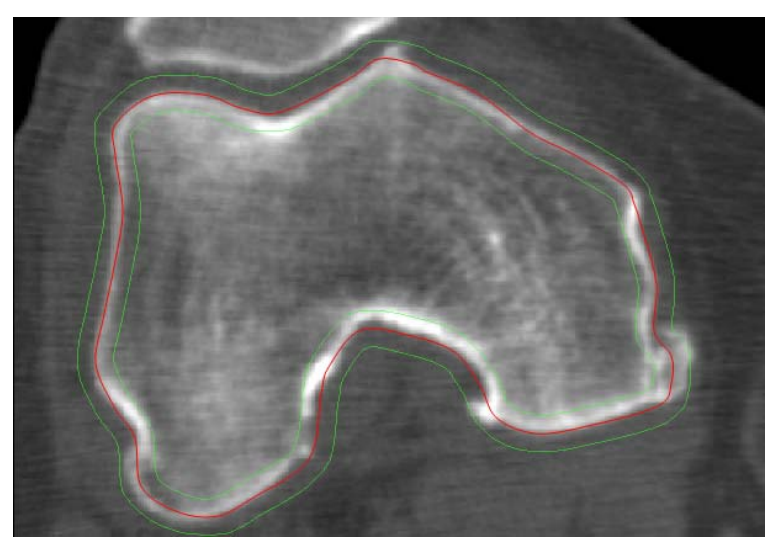

Image 4 shape model (red) registered to image data, and boundary segment region (green) for fine-segmentation.

The statistical model (based on the first 5 modes) gives the variability of the bones in good accordance (see Image 4), so that with a registration of the model with the image data a rough segmentation can be done: In a first step, the initials-position and the scale is searched in the image data set, by doing the best fit with the average model in the volume data (a coarse grid following). Then an iterative model adaptation [3] is made by using the first five shape variations.

With the registered model, we obtain on one hand the model surface and on the other hand the model parameters. The surface data are the bone segments, which we convert for further processing into binary image data $\left\{\Omega_{k}\right\}$. The model parameters of knee joint bones together with those of hip- and ankle-joint - serve as a basis of planning for the determination of the leg axis.

\section{$2.2 \quad$ Fine-Segmentation}

With the fine segmentation, the local nuances of the bone surface should be as precisely as possible. For that we use the front-propagation method - Fast Marching - and scan the bones from the outside (in the boundary region of the segment $\Omega_{k}$ ). The initial front (or the seed-points) is placed outside of the segment $\Omega_{k}$ by the measure $d_{\text {out }} \approx 7$ $\mathrm{mm}$. The front propagation is then given by a speed function, which is calculated from the local image data. This approach is in some sense comparable with the region growing process, but it is better to control because of the cumulative behavior (in terms of the impact values) and therefore more robust. In addition, we use for the control of the front propagation a maximum depth of penetration $d_{\text {in }}$. Is this measure through the front propagation exceeded, it will be reported as a local segmentation error. And after the Fast Marching method there are applied some morphological post-processing steps: on one hand for the blob filtering and the optimization of the segment boundary and on the other hand for the correction of possible segmentation failures.

\subsubsection{Fast-Marching-Algorithm}

The Fast Marching algorithm describes the frontpropagation behavior [5] to a monotonically spread front $C$, i.e. the determining speed function $F(\mathbf{x})$ (defined over the image domain) is always positive $F(\mathbf{x}) \geq 0$. If the front $C$ is implicitly described by a level-set-function $C(t)$ $=\left\{\mathbf{x} \in \mathbf{R}^{n} \mid \phi(\mathbf{x}, t)=0\right\}$, then this function $\phi$ is determined by the level set equation

$$
\phi_{t}+F|\nabla \phi|=0
$$

and by the initial conditions $\phi(\mathbf{x}, 0)=\phi_{0}(\mathbf{x})$, and $F(\mathbf{x})$ is the speed function. Since the front propagation should stop or decrease in the area of edges, it can be expressed by

$$
F(\mathbf{x})=1 /(1+|\nabla f(\mathbf{x})|)
$$

or by a sigmoid function, where $f(\mathbf{x})$ is the value of the pixel $\mathbf{x}$. The segmentation is then given with $\Omega(t)=\{\mathbf{x} \in$ $\left.\mathbf{R}^{n} \mid \phi(\mathbf{x}, \infty) \leq t\right\}$, in which $t$ is a positive parameter. 
For the interpretation of the setting parameter $t$ and a better understanding of the propagation field $T(\mathbf{x})$ := $\phi(\mathbf{x}, \infty)$, the Eikonal equation

$$
|\nabla T(\mathbf{x})|=1 / F(\mathbf{x})
$$

with the boundary conditions $T\left(\mathbf{x}_{0}\right)=0$ (for the seed points $\mathbf{x}_{0}$ ) is more suitable. Then the linked Fermat's principle states [6] that $T(\mathbf{x})$ describes the minimal effort in the field $1 / F(\mathbf{x})$ for moving from point $\mathbf{x}_{0}$ to $\mathbf{x}$. If for the speed function the expression (2) is used, then it can be shown, that $T(\mathbf{x})$ is basically a minimal accumulation of the gradient magnitudes $|\nabla f(\mathbf{x})|$ on a path from $\mathbf{x}_{0}$ to $\mathbf{x}$. And this is one of the advantages of this approach - compared with the region growing algorithm.

By setting a threshold $t>0$ - simplified considered as a measure of the expected accumulation of gradient magnitudes - and knowing the propagation field $T(\mathbf{x})-$ provide by the Fast Marching method -, the fine segmentation is given by

$$
\Omega_{k}^{\prime}=\left\{\mathbf{x} \in \mathbf{R}^{n} \mid T(\mathbf{x}) \leq t\right\} .
$$

The image 5 (left) shows the propagation field $T(\mathbf{x})$, with the seed points $\mathbf{x}_{0}$ shifted to the outside of $\Omega_{k}$ by 15 pixels, in pseudo colors from blue $(T=0)$ to red ( $T \geq$ 100). And one can also recognize a region with low boneedges (cyan to yellow), but due to the accumulation of the low gradient magnitudes, a good segmentation is still possible. Image 5 (right) shows the segment boundary with a threshold value $t=30$.
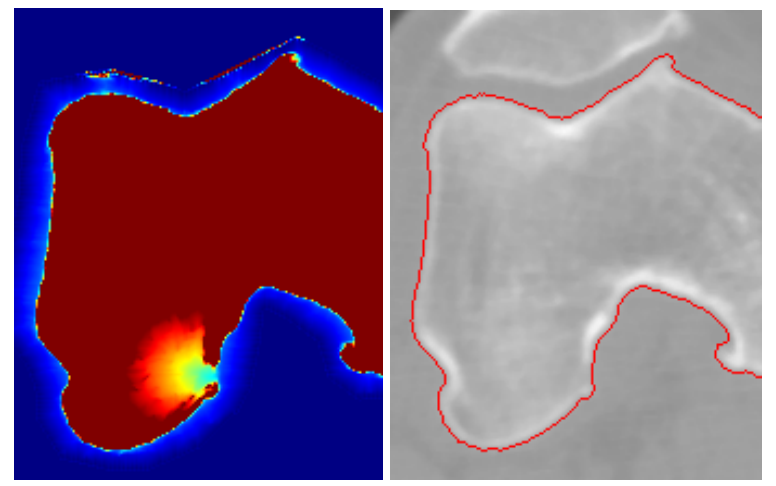

Image 5 Fine-segmentation with Fast-Marching: left the propagation field, right the segment boundary with $t=30$.

In our work we have used for the speed function the sigmoid function. On one hand this allows more influence, but on the other hand it makes the determination of the setting parameter more difficult.

\subsubsection{Postprocessing}

According to the Fast Marching algorithm $\Omega_{k} \rightarrow \Omega^{\prime}{ }_{k}, \Omega^{\prime}{ }_{k}$ may not be simply connected and can contain additional small blob's: e.g. because of noise or the positioning of seed points inside other segments. These artifacts can be removed using morphological operations. Further possible errors (exceeding of the penetration depth) are eliminated with the watershed method.

\section{$3 \quad$ Results}

We have developed a hybrid segmentation method for the knee-bone segmentation from CT data, which is stable and has a good accuracy. A model-based approach (as a pre-segmentation) guarantees a robust separation of femur, tibia and patella. And with the local acting front propagation method Fast Marching (as a fine segmentation) there will be generally automatic achieved an improvement of the segmentation accuracy (respectively it will capture more surface details). But for image data with locally very weak bone edges, segmentation failures can occur, which are recognized by certain criteria's, and they can be easily corrected by changing local parameters.

To validate the accuracy, 20 data sets were segmented manually and with the presented approach: without the fine segmentation the mean deviation is $2.2 \mathrm{~mm}$, with the fine-segmentation it is $0.8 \mathrm{~mm}$, and by some manual corrections the mean value is $0.3 \mathrm{~mm}$.

\section{References}

[1] Ng, V.Y., DeClaire, J.H., Berend, K.R., Gulick, B.C., Lombardi, A.V.: Improved accuracy of alignment with patientspecific positioning guides compared with manual instrumentation in TKA. Jr., Clin Orthop Relat Res. 2012; 470(1):99-107.

[2] Noble, J.W., Moore, C.A., Liu N.: The value of patient-matched instrumentation in total knee arthroplasty. J Arthroplasty, $2012 ; 27(1): \overline{153}-5$.

[3] Cootes, T.F., Taylor, C.J., Cooper D.H., and Graham, J.: Active shape models - their training and application. Computer Vision and Image Understanding, 1995; 61(1):38-59.

[4] Heimann, T., Meinzer, H.P.: Statistical shape models for 3D medical image segmentation: A review. Medical Image Analysis, 2009; 13(4): 543-563.

[5] Sethian, J.A.: Level Set Methods and Fast Marching Methods: Evolving Interfaces in Computational Geometry, Fluid Mechanics, Computer Vision, and Materials Science. Cambridge University Press, 1999.

[6] Cohen, L.: Minimal Paths and Fast Marching Methods for Image Analysis: Handbook of mathematical Models in Computer Vision. Springer 2006. 